\title{
Are we geared up for Medical Audit? - An endeavour for improvement of Quality in Health Care
}

\author{
Dr. N. Lakshmi Bhaskar ${ }^{1}$, Dr. S. Naga Satish $\operatorname{Kumar}^{2}$, M. Meera Subhashini ${ }^{3}$, \\ Dr. K. T. Reddy ${ }^{4}$, Dr. N. Satyanarayana ${ }^{5}$ \\ ${ }^{\prime}$ M.D. (H.A.), Assistant Professor, Department of Hospital Administration, Nizam's Institute of Medical \\ Sciences \\ ${ }^{2}$ M.D. (H.A.), Junior Resident, Department of Hospital Administration, Nizam's Institute of Medical Sciences \\ ${ }^{3}$ BPT, M.H.M., Department of Hospital Administration, Nizam's Institute of Medical Sciences \\ ${ }^{4}$ M.D. (H.A.), Addl. Professor, Department of Hospital Administration, Nizam's Institute of Medical Sciences \\ ${ }^{5}$ M.D. (H.A.), Professor \& HOD, Department of Hospital Administration, Nizam's Institute of Medical Sciences
}

\begin{abstract}
The introduction of quality assurance and medical audit has been an important development in general practice. The present study describes the pros and cons of implementing Medical Audit in Nizam's institute of Medical Sciences, a tertiary care hospital in India, by conducting opinion survey among the faculty members of the institute through a structured questionnaire. The study reveals that majority of doctors were interested in implementing system of Medical Audit through a Medical Audit Committee membered by internal staff with periodical reviews and reforms in policies and guidelines for auditing. The study also revealed that a few proportion of doctors were reluctant to adopt the new system adhering to the traditional thinking and felt medical audit can be a tool of criticism.
\end{abstract}

Keywords: Quality assurance, Medical audit, Quality Health Care

\section{Introduction}

Implementation of systematic quality assurance and medical audit programmes has become a prime issue in many hospitals in view of increasing standards of treatment and patient care. Doctors need to know how to use quality assurance and audit methods, and to see them as feasible and valuable for their work $^{1}$. Studies conducted in Australia, the United Kingdom and the Netherlands found that most doctors had a positive attitude to systematic quality assurance or medical audit in general ${ }^{5,6}$. However, some important obstacles have been identified, such as lack of time, fear of abuse of the audit results by insurers or managers, lack of knowledge of the methodology, little perceived benefit for care providers or patients, inadequate methods for data collection or insufficient use of available data, and the view that quality assurance is boring ${ }^{3,4}$.

According to Peter Hawlock (1993), in implementing of medical audit ${ }^{2}$, there is always some resistance offered against implementation of Medical Audit due to factors like traditional attitude of doctors reluctant to change and negative attitude towards auditing as fault finding method $^{8}$. This can be overcome by the principle that, good points would be drawn first emphasising strengths and the weaknesses will be reviewed internally and deficiencies will be discussed only in context of a constructive plan for change.

Nizam's Institute of Medical Sciences being a renowned super-specialty hospital, people expect a high quality of health care, which can be made possible analyzing the process of providing care through regular medical audits. A minor form of medical audit is being conducted in the institute confined to death cases as Mortality Audit, every fortnightly. Based on this view the present study was conducted to evaluate the possibilities of implementing medical audit, through an opinion survey conducted among faculty doctors of the Institute.

\section{Aim Of The Study:}

To understand the feasibility of implementing Medical Audit in a tertiary care hospital describing the supporting and opposing factors associated.

\section{Objectives:}

1. To assess the opinion of faculty doctors, through a structured questionnaire in regarding implementation of Medical Audit.

2. To understanding driving and restraining factors (strengths and weaknesses) associated with implementing of Medical Audit. 


\section{Methods And Methodology:}

An opinion study was conducted among 60 faculty doctors of various specialties of Nizam's Institute of Medical Sciences through a structured questionnaire containing 9 questions. Faculty Opinion was sort out regarding the answer provided by them to assess various factors affecting in the institute. A descriptive statistic study was adopted using graphical representations of the data.

\section{Observations And Results:}

The following observations were found on reviewing the questionnaire given to the faculty doctors.

Table 1: Observations

\begin{tabular}{|l|l|l|l|}
\hline S. No & Questions & Yes & No \\
\hline 1 & $\begin{array}{l}\text { Do you think that conducting medical audit is required in the present system of } \\
\text { our hospital? }\end{array}$ & 86 & 14 \\
\hline 2 & Are you following audit system in your department? & 0 & 100 \\
\hline 3 & Has your department set any standards for patient care? & 81 & 19 \\
\hline 4 & Do you feel that setting standards is difficult? & 78 & 22 \\
\hline 5 & $\begin{array}{l}\text { Is the information available in our medical records sufficient for conducting } \\
\text { medical audit? }\end{array}$ & 8 & 92 \\
\hline
\end{tabular}

Venn diagram: Perceived Objectives of Medical Audit by the Faculty (According to you, the objective of medical audit is: a. Standardization of treatment, $b$. To give better patient care in future, c. To highlight deficiencies, d. Depends on who is being and who audits)

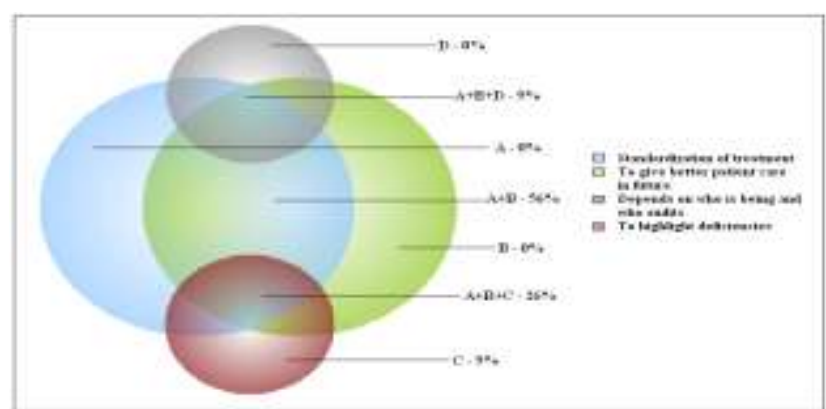

Pie Chart 1: Frequency of Medical Audit (At what frequency should the medical audit be conducted?)

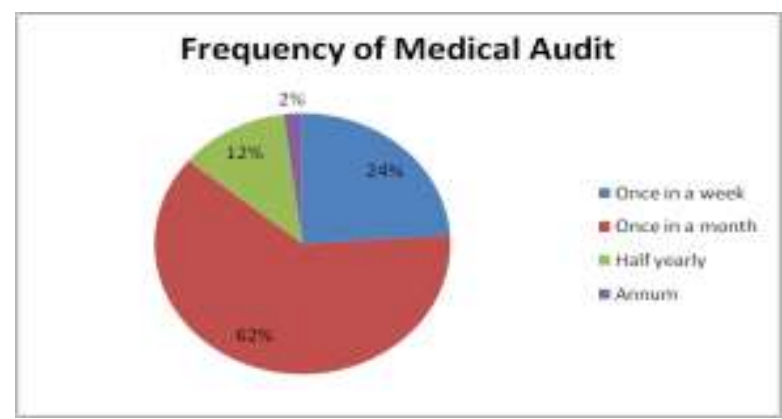

Pie Chart 2: System of Medical Audit (who should conduct medical audit?)

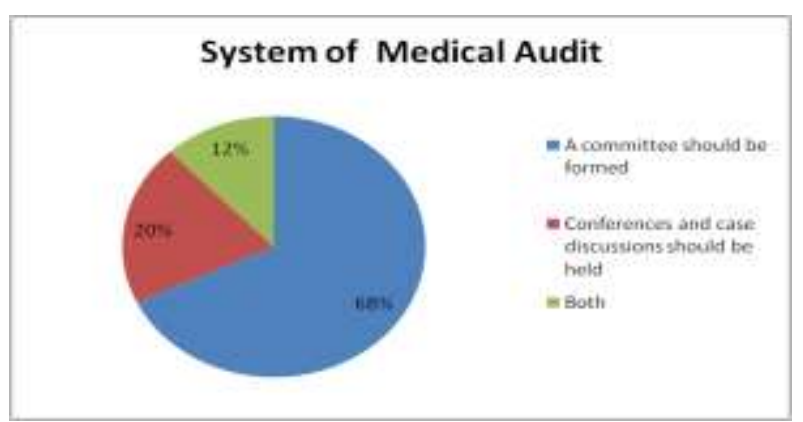

Pie Chart 3: Committee Composition (who should make the team of audit committee?) 


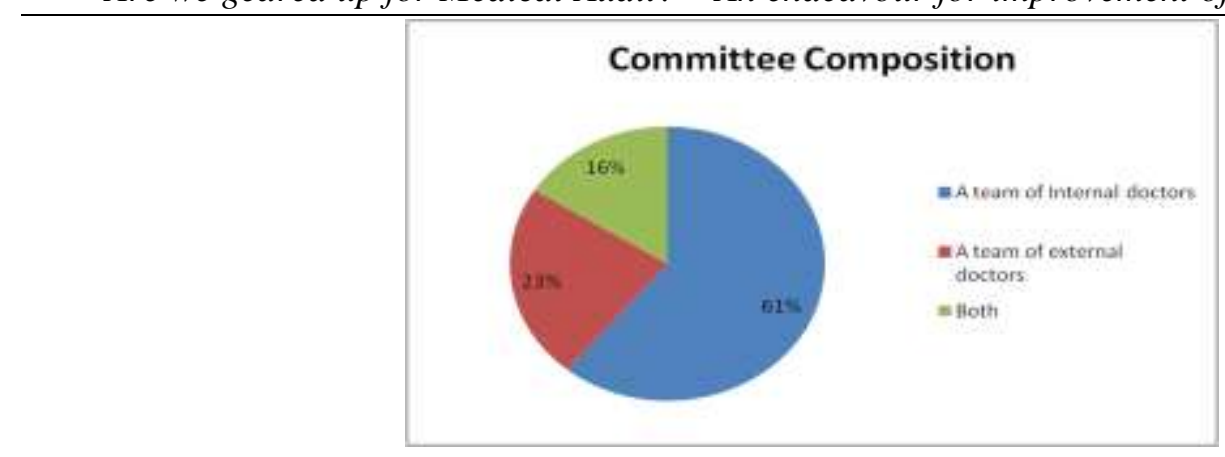

On reviewing the questionnaire along with the explanations given by the faculty doctors, it can be noticed that $86 \%$ and $16 \%$ of faculty doctors voted for and against conducting Medical Audit in the institute respectively. The faculty who voted against were asked to give reasons, of which major reasons were feeling of inertia to change, as the present system is perfect, Medical Records were not adequate for auditing, individual ego and fear of criticism by others about fault finding in their treatment.

As much as $62 \%$ of faculty doctors opined that medical audit should be conducted every monthly, whereas $24 \%$ and $12 \%$ of faculty doctors opined auditing every weekly and half yearly respectively. Very few i.e. $2 \%$ of faculty doctors opined to have auditing per annum. When the questionnaire papers were reviewed these $2 \%$ of doctors were subset of faculty doctors who opted out for medical audit.

Objectives of medical audit were perceived variedly among the faculty doctors. Majority of the faculty doctors around $82 \%$ felt that the objectives of medical audit were to improve standard of treatment for a better patient care. Few faculty doctors around $9 \%$ felt that objective could be biased between the auditor and the department or person who is being audited. $9 \%$ of faculty doctors felt that medical audit can be a tool to highlight the deficiencies in their treatment and of hospitals; in turn can lead to defame the hospital.

It can be noticed that none of the departments in the institute conduct their own medical audits. The faculty doctors explained that work pressure, lack of time, lack of belief in the system of medical audit were the main reasons that prevented implanting their own medical audit system. $81 \%$ of faculty doctors reported that their department has preset standards for patient care, but almost informed that these standards were followed as instructions from the superior but were not in written format. $78 \%$ of faculty doctors agreed that setting standards and following them is not difficult. The rest justified by giving the reason that setting standards would be difficult as condition varies from patient to patient in a same disease.

Majority of the faculty doctors around $68 \%$ opted for formation of committee rather than an open conference or case discussion. They felt that treatment varies from doctor to doctor and open discussion might point out the mistakes of the treating doctor. $20 \%$ of faculty opted open conferences and case discussion in fear of chance of biased decision made by the committee. The rest opted for both to eliminate the disadvantages of both. $61 \%$ of doctors wished internal doctors compose the audit team, as the institute has good number of experienced and unbiased faculty and felt that external doctors might expose the loopholes and defame the institute. It was surprising to note that $92 \%$ of faculty doctors felt that the information present in the medical records was insufficient for conducting medical audit in the institute.

Few doctors suggested that a rigorous support from top level management and well planned implementation from middle level management with involvement of each member of the organisation can make medical audit success, thereby providing quality health care.

\section{Discussion:}

It is surprising to know that $14 \%$ of faculty were against the concept of implantation of medical audit in the institute. Most of the faculty felt that existing medical records weren't sufficient for conducting medical audit. Moreover no practice of medical audits in individual departments and negative attitude towards system of medical audit make the environment improper for implementation of medical audits. This can be overcomed first by identifying the strengths likes willingness of majority of doctors, competition for quality, defending from Consumer protection act, increased need for accreditation, etc and weaknesses like traditional thinking of doctors, incomplete and inaccurate medical records, egoistic attitudes of faculty, misinterpreting medical audit as fault finding technique, etc $^{8}$. Later strengthening the supporting factors and eliminating weaknesses, by various methods like proper orientation, communication, participation and involvement of the members of the institute and facilitation and support from the management ${ }^{9,10}$. The system should be implemented in a planned manned manner involving every faculty of the institute, in a step wise pattern. Audit should be implemented in individual departments initially, extending to whole hospital slowly, refining and redefining the protocols and guidelines. Once the system of auditing has been established, regular reviews should be considered to revise the system to tackle the hurdles encountered. 
The following method as suggested from the present study can be adopted to implement medical audit in a hospital:

- Every department should plan for their own audit process and submit the plan to higher authorities or top level management.

- All set standards or protocols must be in written form and periodically reviewed and revised to set up higher standards.

- The system should be introduced and every faculty must be oriented and trained in maintaining standards.

- Senior doctors must verify medical records regularly and check for completeness of records. They must train their juniors and naïve doctors in marinating a proper medical record.

- Medical record department should check for incomplete medical records and periodically submit their report to higher authorities and top level management.

- Medical audit committee must be formed by experienced, unbiased internal doctors with representations from clinical departments, pre-clinical and para-clinical departments, hospital administration and nursing administration. The committee should regularly audit quality of patient care through medical records.

- Regularly, usually annually medical audit must be conducted by external members like NABH, India, to control and relate system of audit and help in improving quality health care and increasing standards for patient care and hospital.

\section{Conclusion:}

Medical audits if utilised efficiently and rigorously help the hospital to perform better both for itself and its patients, but needs acceptability by the hospital systems and medical fraternity as an improvement initiative rather than a fault finding mechanism ${ }^{11}$. Well-designed programmes for the implementation of medical audit, using a variety of different interventions, have to be developed. Each member of the organisation must be involved with a positive attitude towards implanting medical audit, ensuring satisfactory results in providing quality health care to every patient.

\section{References:}

[1] Lawrence M. What is medical audit? In: Lawrence M, Schfield T, editors. Medical Audit in Primary Health Care. New York: Oxford University Press; 1993.

[2] Fraser R, Lackani M, Baker R. Evidence-Based Clinical Audit. 1st ed. Oxford: Butterworth- Heinemann; 1998.

[3] Marinker M. Principles in Medical Audit and General Practice. London: BMJ Publishing Group; 1990.

[4] RCGP. Information sheet module10. Clinical Audit in General Practice. 2002 Jan. Available from: URL: www.gpnetwork.net.au/eduseru/10- keyiss.htm/.

[5] Sheldon MG. Audit in General Practice. Practice Update. 1989; 5:1052.

[6] RCGP. Information sheet No.17. Clinical Audit in General Practice. 2002 Feb. Available from: URL: www.gpnetwork.net.au/eduseru/1 - backgr.htm/.

[7] Webb S, Dowell A, Heywood P. Survey of general practice audit in Leeds. BMJ 1991; 302: 390-392.

[8] Black N, Thompson E. Obstacles to medical audit: British doctors speak. Soc Sci Med 1993; 36: 849-856.

[9] Morrison J, Sullivan F. Audit: teaching medical students in general practice. Med Educ 1993; 27: 495-502.

[10] Newton J, Hutchinson A, Steen N, et al. Educational potential of medical audit: observations from a study of small group setting standards. Qual Health Care 1992; 1: 256-259.

[11] North of England study of standards and performance in general practice. Medical audit in general practice. I: Effects on doctors' clinical behaviour for common childhood conditions. BMJ 1992; 304: 1480-1484. 